\title{
Missed Work Related to Mid-Week Screening Colonoscopy
}

\author{
Mamie H. Dong $\cdot$ Denise Kalmaz $\cdot$ Thomas J. Savides
}

Received: 5 October 2010/Accepted: 18 December 2010/Published online: 8 January 2011

(c) The Author(s) 2011. This article is published with open access at Springerlink.com

\begin{abstract}
Background Most screening colonoscopies require patients to miss work on the day of the procedure. Little is known about whether patients are taking additional time off from work, and the reasons for doing so.

Aims The purpose of this study was to assess the patterns and reasons for missed work related to screening colonoscopies.

Methods All outpatient screening colonoscopy procedures performed at an academic medical center over 6 months were reviewed. Exclusions included procedures performed for other indications, patients age 65 or older, procedures performed on Monday or Friday, and patients who were not working. Patients were interviewed by telephone regarding missed work time and the reasons for doing so.

Results Sixty-eight patients met all inclusion criteria. Thirty-four percent missed work on more than the day of the procedure. Thirty-two percent took the day prior off, $10 \%$ took the day after off, and $9 \%$ took both days off. The reason for taking the day before the procedure off was uniformly anticipation of the bowel preparation. Of those who took the day after off, $57 \%$ did so as a precautionary measure after moderate sedation, while $43 \%$ had symptoms.
\end{abstract}

\author{
M. H. Dong $(\bowtie) \cdot$ D. Kalmaz · T. J. Savides \\ Division of Gastroenterology, University of California at San \\ Diego, 9500 Gilman Drive MC 0063, La Jolla, CA 92093, USA \\ e-mail: mhdong@ucsd.edu \\ D. Kalmaz \\ e-mail: dkalmaz@ucsd.edu \\ T. J. Savides \\ e-mail: tsavides@ucsd.edu
}

Conclusions One third of working patients who undergo mid-week screening colonoscopies miss work on additional days to the procedure day. Unanticipated time missed from work could increase the indirect costs of screening colonoscopy.

Keywords Colonoscopy $\cdot$ Screening · Health care costs $\cdot$ Lost productivity
Abbreviations
CT Computer tomography
EGD Esophagogastroduodenoscopy
GI Gastroenterology
IBD Inflammatory bowel disease
IBS Irritable bowel syndrome

\section{Introduction}

Patients undergoing screening colonoscopy typically miss work on the day of their procedure due to both the procedure itself and to the sedation, but little is known about what the recovery time is after the procedure, whether many patients miss work the day before the procedure, and whether a significant number of friends and family members miss work as a result of a patient's colonoscopy procedure. In a study of subjects undergoing screening or surveillance colonoscopies, Ko et al. [1] interviewed 502 patients aged 40 years and older, and reported that $20 \%$ of them lost more than 1 day from normal activities and $25 \%$ of friends or family lost at least 1 day. Jonas et al. [2, 3] examined time requirements for screening colonoscopy in 110 subjects and found that the average total time required, 
from initiation of the bowel preparation to return to normal activities, was $39.5 \mathrm{~h}$, with $17 \%$ of subjects taking longer than $24 \mathrm{~h}$ to achieve full recovery. These studies included patients who had procedures any day of the week, Monday through Friday. Also, they examined time lost from self reported "normal activities," both work-related and nonwork related. It is anticipated that the number of missed days from work would be even higher if procedures were performed mid-week (Tuesday, Wednesday, or Thursday) because no weekend (Saturday/Sunday) days would be missed from work. The main aim of this paper was to determine the frequency of missing work the day before or the day after screening colonoscopy when performed midweek.

\section{Methods}

This was a retrospective study conducted at the University of California at San Diego Medical Center. All outpatient colonoscopies performed at the UCSD Thornton Hospital GI endoscopy unit, UCSD Moores Cancer Center endoscopy unit, or UCSD University Ambulatory Surgery Center within a 6-month time period (from August 2009 through January 2010) were reviewed for eligibility by examining an electronic endoscopy report database.

As per institutional practice at the time, all patients were instructed to consume a clear liquid diet the day before the procedure, followed by 41 of polyethylene glycol solution starting at $6 \mathrm{pm}$ the evening before the procedure. All patients were instructed that they needed to have a friend or family member accompany them home after the procedure if they received intravenous sedation.

Inclusion criteria were: (1) adults under the age of 65 , (2) colonoscopies performed in asymptomatic individuals for the indications of average risk screening, surveillance in individuals with personal history of adenomatous polyps or colon cancer, screening in individuals with high risk family history, evaluation after other abnormal screening test such as flexible sigmoidoscopy, barium enema, or fecal occult blood test, and for evaluation of iron deficiency without overt GI bleeding, and (3) procedures scheduled for the middle of the week (Tuesday, Wednesday, or Thursday), flanked by working days before and after the procedure.

Exclusion criteria were: (1) subjects who were 65 years of age and older based on the United States Social Security Office established standard retirement age, (2) those who had procedures performed for evaluation of overt GI bleeding, abdominal pain or bloating, change in bowel habits, as a follow-up procedure to remove a known lesion, or for evaluation of abnormal imaging findings (excluding imaging studies performed for the purposes of colon cancer screening), (3) procedures performed in subjects with inflammatory bowel disease (IBD), and (4) procedures performed on Mondays, Fridays, and immediately adjacent to national holidays as subjects would normally be expected to have either the day before or after the procedure off.

Those that met inclusion criteria were contacted by telephone, and those who agreed to participate were asked a series of questions regarding whether they had missed work before or after their procedure, the reasons for missing work, and whether any friends or family took time off of work for their procedure. Work was self-defined by the patient as having a conventional job, being self employed, working from home, or being contracted. Subjects who had colonoscopies scheduled during vacation days, or subjects in the latter three groups who did not have active work-related tasks on the days surrounding their procedure were classified as working but not requiring time off. Subjects were also asked to estimate the number of sick days they took within the past year (excluding days taken for the colonoscopy procedure) and whether they had a known diagnosis of irritable bowel syndrome (IBS). Ethnicity was self-reported. All telephone calls were made by a single investigator (MHD). Subjects who could not be reached on three separate days (at least one call was made on a weekday evening between 7 and 9 pm, and another on a weekend afternoon between 1 and 4 pm) were excluded from the study. For those that reported not working during the month of their colonoscopy procedure, information was collected regarding whether friends or family members missed work.

Chart reviews were conducted to obtain the following information: patient age, sex, exam indication, endoscopist, procedure time (morning vs. afternoon), sedative medication regimens and dosages, endoscopic interventions performed, whether or not the exam was completed to the cecum, and whether any other concomitant endoscopic procedures were performed that day (i.e., EGD).

This study was approved by the Human Research Patient Protection Institutional Review Board of the University of California, San Diego. No external funding sources were utilized.

\section{Results}

\section{Subject Characteristics}

A total of 799 outpatient colonoscopies were performed from August 2009 through January 2010. The reasons for excluding 687 were: 234 were performed for purposes other than screening or surveillance, 286 were performed on Mondays, Fridays, or immediately adjacent to national holidays, 92 were performed in subjects age 65 or older, 16 
declined to participate, and 59 could not be reached by telephone. A total of 112 subjects participated in the study. Of those, 44 reported not working during the month of their colonoscopy procedure, while 68 were working (Fig. 1).

Characteristics of the 68 working subjects are shown in Table 1. The mean age was 53.9 years (range
40-64 years), 56\% were women, and the majority of the group was Caucasian (79\%). Very few reported having a formal diagnosis of IBS (4\%). For the patients who were not working, the mean age was 56.8 years (range 43-64 years), $66 \%$ were women, $82 \%$ were Caucasian, and $2 \%$ reported a diagnosis of IBS (Table 1). Procedure

Fig. 1 Patient recruitment

\section{Outpatient Colonoscopies Performed}

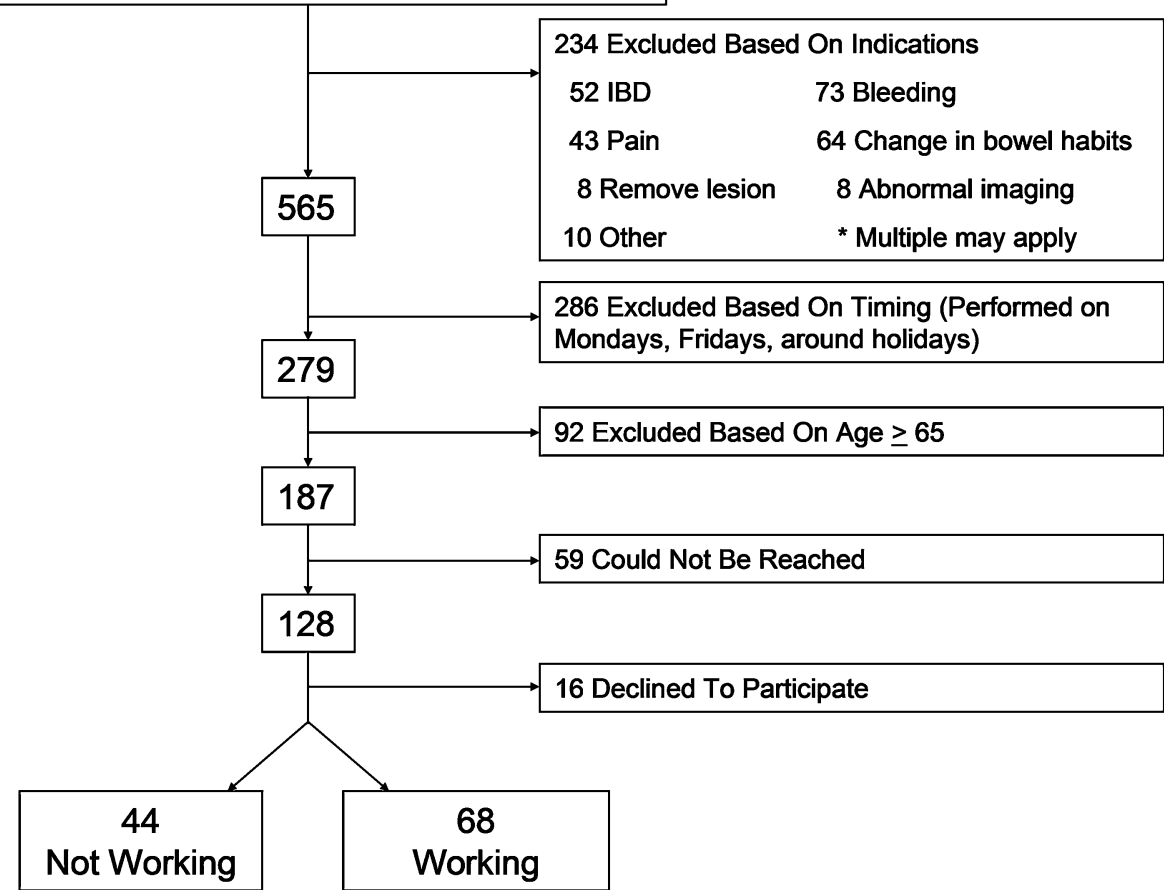

Table 1 Patient characteristics

\begin{tabular}{lcc}
\hline Characteristics & Working group $(N=68)$ & Non-working group $(N=44)$ \\
\hline Mean age, y (range) & $53.9(40-64)$ & $56.8(43-64)$ \\
Women & $38(56 \%)$ & $29(66 \%)$ \\
Previous diagnosis of IBS & $3(4 \%)$ & $1(2 \%)$ \\
Caucasian ethnicity & $54(79 \%)$ & $36(82 \%)$ \\
Morning procedures & $41(60 \%)$ & $26(59 \%)$ \\
Had concomitant endoscopic procedures & $4(6 \%)$ & $7(16 \%)$ \\
Indication for procedure & & $32(73 \%)$ \\
Average risk screening & $45(66 \%)$ & $4(9 \%)$ \\
High risk family history & $10(15 \%)$ & $8(18 \%)$ \\
History of polyps or colon cancer & $11(16 \%)$ & $0(0 \%)$ \\
Iron deficiency anemia & $2(3 \%)$ & $4.0(0-6)$ \\
Procedure sedation & & $85(0-150)$ \\
Mean midazolam dose, mg (range) & $4.0(0-8)$ & $72(0-100)$ \\
Mean meperidine dose, mg (range) & $90(0-150)$ & \\
Mean fentanyl dose, mcg (range) & $50(0-100)$ & $25(57 \%)$ \\
Procedure interventions & & $17(39 \%)$ \\
None & $35(52 \%)$ & $4(9 \%)$ \\
Biopsy forceps & $23(34 \%)$ &
\end{tabular}


Table 2 Missed work related to colonoscopy procedures

\begin{tabular}{lcl}
\hline Missed work & $\begin{array}{l}\text { Working group } \\
(N=68)\end{array}$ & $\begin{array}{l}\text { Non-working group } \\
(N=44)\end{array}$ \\
\hline Patients who took days off from work & $23(34 \%)$ & NA \\
Took the day prior off & $22(32 \%)$ & NA \\
In anticipation of the prep & $22(100 \%)$ & NA \\
Took the day after off & $7(10 \%)$ & NA \\
Abdominal pain & $1(14 \%)$ & NA \\
Altered hearing & $1(14 \%)$ & NA \\
Sore throat & $1(14 \%)$ & NA \\
Anticipatory & $4(57 \%)$ & NA \\
Took both the day before and the day after off & $6(9 \%)$ & NA \\
Friends or family who took days off from work & $33(49 \%)$ & $18(41 \%)$ \\
Half to one day & $32(47 \%)$ & $18(41 \%)$ \\
Two days & $1(2 \%)$ & 0 \\
Average number of sick days taken in the prior year & $2.1($ range $0-10)$ & NA \\
\hline
\end{tabular}

NA not applicable

${ }^{\text {a }}$ Excluding two outliers who took 150 and 23 days off for major illness someone drive or accompany the patient to their home after sedation. One family member took 2 days off work because the patient had a slow recovery after colonoscopy. In the non-working group, $18(41 \%)$ reported that a friend or family member took time off of work. All were the designated drivers for the procedure. Combining the two groups, $46 \%$ of patients (51 of 112) had friends or family members who had to miss work as a result of the colonoscopy procedure (Table 2).

\section{Discussion}

This study found that among asymptomatic, working individuals who had screening or surveillance colonoscopies performed on a Tuesday, Wednesday, or Thursday, $34 \%$ missed work on more than the day of the procedure. Thirty-two percent took the day prior off, $10 \%$ took the day after off, and $9 \%$ took both days off. Forty-six percent of individuals had friends or family members who also took time off work for the procedure. These figures are somewhat higher than the $20 \%$ incidence of two or more patient days lost and $25 \%$ incidence of one or more family days lost from normal activities previously reported by Ko et al. [1]. However, their study population included all patients undergoing screening and surveillance colonoscopies and reported time lost from normal activities, whereas ours included only those who were working at the time of the procedure, had procedures performed mid-week, and specifically examined lost work time.

In our study population, a high proportion of patients $(32 \%)$ took the day prior to the procedure off in anticipation of the bowel preparation, even though the instructions they were given did not require them to start consuming the polyethylene glycol solution until the evening before the 
Table 3 Comparison of those who missed work versus those who did not $(N=68)$

\begin{tabular}{|c|c|c|c|c|}
\hline Characteristic & $\begin{array}{l}\text { Missed any work } \\
(N=23)\end{array}$ & $\begin{array}{l}\text { Missed the day } \\
\text { before }(N=22)\end{array}$ & $\begin{array}{l}\text { Missed the day } \\
\text { after }(N=7)\end{array}$ & $\begin{array}{l}\text { Did not miss work } \\
(N=45)\end{array}$ \\
\hline Mean age, y (range) & $52.7(40-64)$ & $52.6(40-64)$ & $53.1(51-56)$ & $54.5(44-64)$ \\
\hline Women & $12(52 \%)$ & $12(55 \%)$ & $3(43 \%)$ & $26(58 \%)$ \\
\hline Previous diagnosis of IBS & $1(4 \%)$ & $1(5 \%)$ & $0(0 \%)$ & $2(4 \%)$ \\
\hline Caucasian ethnicity & $19(83 \%)$ & $18(82 \%)$ & $4(57 \%)$ & $35(78 \%)$ \\
\hline Morning procedures & $12(52 \%)$ & $12(55 \%)$ & $4(57 \%)$ & $29(64 \%)$ \\
\hline Had concomitant endoscopic procedures & $2(9 \%)$ & $2(9 \%)$ & $2(29 \%)$ & $2(4 \%)$ \\
\hline \multicolumn{5}{|l|}{ Indication for procedure } \\
\hline Average risk screening & $15(65 \%)$ & $14(64 \%)$ & $7(100 \%)$ & $30(67 \%)$ \\
\hline High risk family history & $3(13 \%)$ & $3(14 \%)$ & $0(0 \%)$ & $7(16 \%)$ \\
\hline History of polyps or colon cancer & $4(17 \%)$ & $4(18 \%)$ & $0(0 \%)$ & $7(16 \%)$ \\
\hline Iron deficiency anemia & $1(4 \%)$ & $1(5 \%)$ & $0(0 \%)$ & $1(2 \%)$ \\
\hline \multicolumn{5}{|l|}{ Procedure sedation } \\
\hline Mean midazolam dose, mg (range) & $3.9(0-8)$ & $4.0(0-8)$ & $3.4(3-4)$ & $4.1(0-7)$ \\
\hline Mean meperidine dose, mg (range) & $82(0-100)$ & $81(0-100)$ & $86(75-100)$ & $93(0-150)$ \\
\hline \multicolumn{5}{|l|}{ Procedure interventions } \\
\hline None & $16(70 \%)$ & $16(73 \%)$ & $4(57 \%)$ & $19(42 \%)$ \\
\hline Biopsy forceps & $6(26 \%)$ & $5(23 \%)$ & $2(29 \%)$ & $23(51 \%)$ \\
\hline Snare polypectomy & $3(13 \%)$ & $2(9 \%)$ & $2(29 \%)$ & $7(16 \%)$ \\
\hline
\end{tabular}

IBS irritable bowel syndrome

procedure. It is possible that better pre-procedure instructions and reassurance would have avoided the patients taking the day prior to colonoscopy off work. Ten percent of patients took the day after the procedure off, and more than half of those did so as a precautionary measure rather than in response to true symptoms. Only three patients (4\%) developed symptoms after colonoscopy, and two of those were likely unrelated to the procedure (sore throat and altered hearing). Thus, less than $2 \%$ of patients had symptoms after the colonoscopy procedure, attributable to the procedure itself, which were severe enough to necessitate missing a day of work. In those who had no symptoms but took the day off, anxiety related to the potential after-effects of sedation appears to be the main factor.

Colonoscopy procedures required a significant amount of time investment from friends and family members, with $46 \%$ of patients in our cohort reporting that a friend or relative took time off of work for the procedure. In all but one case, the reason for missing work was due to the requirement that patients not drive themselves home after receiving moderate sedation.

Our patient population was fairly uniform, consisting of mostly middle to upper class Caucasians living in San Diego, California, and may not be generalizable to other geographic or ethnic cohorts. On average, this cohort took only 2.1 sick days off of work in the prior year, suggesting that this is an otherwise fairly healthy and motivated working group. Due to the retrospective nature of the study, subjects may be influenced by recall bias. Also, our study cohort consisted of individuals who had chosen to undergo colonoscopy procedure, and may not represent all individuals eligible for screening or surveillance exams.

In order to assess missed work the day before and after the procedure, only mid-week cases were analyzed. Cases done at the beginning (Mondays) or end (Fridays) of the week would have less impact on patients missing work. From an employer's perspective, this might suggest that more screening colonoscopies should be done on Mondays or Fridays in order to minimize lost work time. This might also be the preference of patients, who might be selfemployed or who might need to use medical or vacation leave for missed work related to the procedures. From a societal perspective, as a rough estimate, if there are 42 million US adults age 50-65 (US Census 2000), with $64.6 \%$ participating in the labor force (US Bureau of Labor Statistics July 2010), an average weekly wage of $\$ 843$ (US Bureau of Labor Statistics, third quarter 2009 for those age 55-65), and 50\% utilization of screening colonoscopy [4], if $34 \%$ of those undergoing screening colonoscopy take one additional day off from work, that would amount to $\$ 778$ million in lost wages. Intervention with patient education about bowel prep techniques and what to expect before and after the procedure might reduce the amount of missed work.

In conclusion, a high percentage of previously asymptomatic patients take more time off of work than the actual 
procedure day for colonoscopies, which is mostly related to anxiety about the procedure process. A significant number of patients also have friends or family members who must take time off of work as a result of the procedure. The indirect financial costs to society related to missed work for screening and surveillance colonoscopies should be taken into account, and efforts should be made to minimize those costs when possible through patient education and reassurance. Further studies are needed to assess missed work related to colonoscopy procedures in different demographic populations, in those with varying work responsibilities, in patients receiving conscious sedation versus propofol, and in those who receive conventional versus split-dose bowel preparations.

Conflict of interest The authors have no conflict of interest or financial disclosures.

Open Access This article is distributed under the terms of the Creative Commons Attribution Noncommercial License which permits any noncommercial use, distribution, and reproduction in any medium, provided the original author(s) and source are credited.

\section{References}

1. Ko CW, Riffle S, Shapiro JA, et al. Incidence of minor complications and time lost from normal activities after screening or surveillance colonoscopy. Gastrointest Endosc. 2007;65: $648-656$.

2. Jonas DE, Russell LB, Sandler RS, Chou J, Pignone M. Patient time requirements for screening colonoscopy. Am J Gastroenterol. 2007; $102: 2401-2410$

3. Jonas DE, Russell LB, Sandler RS, Chou J, Pignone M. Value of patient time invested in the colonoscopy screening process: time requirements for colonoscopy study. Med Decis Mak. 2008;28: $56-65$.

4. CDC (Center for Disease Control). Vital signs: colorectal cancer screening among adults aged 50-75 years-United States, 2008. MMWR 2010; 59:808-812. 